\title{
Positive Solutions for a System of Fractional Integral Boundary Value Problems Involving Hadamard-Type Fractional Derivatives
}

\author{
Haiyan Zhang $\mathbb{D}^{1},{ }^{1}$ Yaohong $\mathrm{Li}\left(\mathbb{D},{ }^{1}\right.$ and Jiafa $\mathrm{Xu} \mathbb{D}^{2}$ \\ ${ }^{1}$ School of Mathematics and Statistics, Suzhou University, Suzhou, Anhui 234000, China \\ ${ }^{2}$ School of Mathematical Sciences, Chongqing Normal University, Chongqing 401331, China \\ Correspondence should be addressed to Jiafa Xu; xujiafa292@sina.com
}

Received 1 July 2019; Accepted 27 August 2019; Published 3 October 2019

Academic Editor: Dimitri Volchenkov

Copyright (C) 2019 Haiyan Zhang et al. This is an open access article distributed under the Creative Commons Attribution License, which permits unrestricted use, distribution, and reproduction in any medium, provided the original work is properly cited.

In this paper, we use fixed-point index to study the existence of positive solutions for a system of Hadamard fractional integral boundary value problems involving nonnegative nonlinearities. By virtue of integral-type Jensen inequalities, some appropriate concave and convex functions are used to depict the coupling behaviors for our nonlinearities $f_{i}(i=1,2)$.

\section{Introduction}

In this paper, we study the existence of positive solutions for the system of Hadamard fractional integral boundary value problems:

$$
\left\{\begin{array}{l}
{ }^{H} D^{\alpha} u(t)=f_{1}(t, u(t), v(t)), \quad t \in(1, e), \\
-{ }^{H} D^{\alpha} v(t)=f_{2}(t, u(t), v(t)), \quad t \in(1, e), \\
u(j)(1)=v^{(j)}(1)=0, \\
u(e)=\int_{1}^{e} h(t) u(t) \frac{d t}{t}, \\
v(e)=\int_{1}^{e} h(t) v(t) \frac{d t}{t}
\end{array}\right.
$$

where $\alpha \in(n-1, n]$ is a real number with $n \geq 3$, $j=0,1,2, \ldots, n-2$, and ${ }^{H} D^{\alpha}$ is the Hadamard fractional derivative. The nonlinearities $f_{i} \in C\left([1, e] \times \mathbb{R}^{+} \times \mathbb{R}^{+}, \mathbb{R}^{+}\right)$, $\mathbb{R}^{+}=[0,+\infty)$. Moreover, the function $h$ on $[1, e]$ satisfies the condition:

$$
\text { (H0) } h \geq 0 \text { with } \int_{1}^{e} h(t)(\log t)^{\alpha-1}(d t / t) \in[0,1) \text {. }
$$

In recent years, the fractional calculus and fractional differential equations are of importance in mathematics, physics, electroanalytical chemistry, capacitor theory, electrical circuits, biology, control theory, and fluid dynamics [1-20]. For example, in [1], the author considered the fractional $(n-1,1)$-type conjugate boundary value problems:

$$
\begin{cases}D_{0+}^{\alpha} u(t)+\lambda f(t, u(t))=0, & 0<t<1, \\ u^{(j)}(0)=0, u(1)=0, & 0 \leq j \leq n-2,\end{cases}
$$

where $\alpha \in(n-1, n], n \geq 3$, and $D_{0+}^{\alpha}$ is the Riemann-Liouville's fractional derivative. By means of Leray-Schauder type and Krasnosel'skii's fixed-point theorems, the author derived an interval of parameter $\lambda$ such that (2) has multiple positive solutions when any $\lambda$ lies in the interval.

On the other hand, we note that coupled systems of fractional differential equations have also been investigated by many authors, see [21-32]. For example, in [21], the authors used a fixed-point theorem of increasing $\varphi$ - $(h, r)$-concave operators to establish the existence and uniqueness of solutions for a system of four-point boundary value problems involving Hadamard fractional derivatives:

$$
\begin{cases}{ }^{H} D^{\alpha} u(t)+f(t, v(t))=l_{f}, & t \in(1, e), \\ { }^{H} D^{\beta} v(t)+g(t, u(t))=l_{g}, & t \in(1, e), \\ u^{(j)}(1)=v^{(j)}(1)=0, & 0 \leq j \leq n-2, \\ u(e)=a v(\xi), & \eta \in(1, e), \\ v(e)=b u(\eta), \xi, & \end{cases}
$$

where $f, g \in C([1, e] \times \mathbb{R}, \mathbb{R})$ and $l_{f}$ and $l_{g}$ are two positive parameters. In [22], the authors established positive 
solutions for the coupled Hadamard fractional integral boundary value problems:

$$
\begin{cases}{ }^{H} D^{\alpha} u(t)+\lambda f(t, u(t), v(t))=0, & t \in(1, e), \lambda>0, \\ { }^{H} D^{\beta} v(t)+\lambda g(t, u(t), v(t))=0, & t \in(1, e), \lambda>0, \\ u^{(j)}(1)=v^{(j)}(1)=0, & 0 \leq j \leq n-2, \\ u(e)=\mu \int_{1}^{e} v(s) \frac{d s}{s}, & \\ v(e)=v \int_{1}^{e} u(s) \frac{d s}{s}, & \end{cases}
$$

where the nonlinearities $f$ and $g$ satisfy either of the following conditions:

$(\mathrm{H})_{\text {Yang1 }}$ : there exists $\left[\theta_{1}, \theta_{2}\right] \subset(1, e)$ such that $\liminf u \longrightarrow+\infty \min _{t \in\left[\theta_{1}, \theta_{2}\right]}(f(t, u, v) / u)=+\infty \quad$ and

$\liminf v \longrightarrow+\infty \min _{t \in\left[\theta_{1}, \theta_{2}\right]}(g(t, u, v) / v)=+\infty$.

$(\mathrm{H})_{\text {Yang2 }}$ : there exists $\left[\theta_{1}, \theta_{2}\right] \subset(1, e)$ such that $\liminf \inf _{v \longrightarrow+\infty} \min _{t \in\left[\theta_{1}, \theta_{2}\right]}(f(t, u, v) / v)=+\infty \quad$ and

$\liminf \operatorname{in}_{u \longrightarrow+\infty} \min _{t \in\left[\theta_{1}, \theta_{2}\right]}(g(t, u, v) / u)=+\infty$.

Inspired by the aforementioned works, in this paper, we use the fixed-point index to consider the existence of positive solutions for system (1) of fractional integral boundary value problems involving Hadamard-type fractional derivatives. Based on integral-type Jensen inequalities, some appropriate concave and convex functions are used to depict the coupling behaviors for the nonlinearities $f_{i}(i=1,2)$. Moreover, our a priori estimates for positive solutions are derived by developing some appropriate nonnegative matrices when $f_{i}(i=1,2)$ grow sublinearly at $\infty$. These conditions here are different from that in $(\mathrm{H})_{\text {Yang1 }}$ and $(\mathrm{H})_{\text {Yang2 }}$.

\section{Preliminaries}

In this paper, we only provide some necessary definitions and lemmas for the Hadamard fractional derivative. For more details about Hadamard fractional calculus, see the book [33].

Definition 1. The Hadamard derivative of fractional order $q$ for a function $g:[1, \infty) \longrightarrow \mathbb{R}$ is defined as

$$
\begin{array}{r}
{ }^{H} D^{q} g(t)=\frac{1}{\Gamma(n-q)}\left(t \frac{d}{d t}\right)^{n} \int_{1}^{t}(\log t-\log s)^{n-q-1} g(s) \frac{d s}{s}, \\
n-1<q<n,
\end{array}
$$

where $n=[q]+1,[q]$ denotes the integer part of the real number $q$, and $\log (\cdot)=\log _{e}(\cdot)$.

Definition 2. The Hadamard fractional integral of order $q$ for a function $g$ is defined as

$$
{ }^{H} I^{q} g(t)=\frac{1}{\Gamma(q)} \int_{1}^{t}(\log t-\log s)^{q-1} g(s) \frac{d s}{s}, \quad q>0 .
$$

Lemma 1. Let $q>0$ and $u \in C[1, \infty) \cap L^{1}[1, \infty)$. Then, the Hadamard fractional differential equation ${ }^{H} D^{q} u(t)=0$ has the solution

$$
u(t)=c_{1}(\log t)^{q-1}+c_{2}(\log t)^{q-2}+\cdots+c_{n}(\log t)^{q-n},
$$

where $c_{i} \in \mathbb{R}, n-1<q<n, n=[q]+1$, and $i=1,2, \ldots, n$.

Lemma 2. Let $q>0$ and $u \in C[1, \infty) \cap L^{1}[1, \infty)$. Then, we have the following formula:

$$
\begin{aligned}
{ }^{H} I^{q H} D^{q} u(t)= & u(t)+c_{1}(\log t)^{q-1}+c_{2}(\log t)^{q-2}+\cdots \\
& +c_{n}(\log t)^{q-n},
\end{aligned}
$$

where $c_{i}$ and $n$ are as in Lemma 1 and $i=1,2, \ldots, n$.

Lemma 3. Suppose that (HO) holds. Let $f \in C[1, e]$. Then, the boundary value problems

$$
\left\{\begin{array}{l}
-{ }^{H} D^{\alpha} u(t)=f(t), \quad t \in(1, e), \\
u^{(j)}(1)=0, \\
u(e)=\int_{1}^{e} h(t) u(t) \frac{d t}{t},
\end{array}\right.
$$

has a unique solution

$$
u(t)=\int_{1}^{e} G(t, s) f(s) \frac{d s}{s},
$$

where

$$
\begin{array}{r}
G(t, s)=G_{1}(t, s)+\frac{(\log t)^{\alpha-1}}{1-\int_{1}^{e} h(t)(\log t)^{\alpha-1} d t / t} \int_{1}^{e} h(t) G_{1}(t, s) \frac{d t}{t}, \\
G_{1}(t, s)=\frac{1}{\Gamma(\alpha)}\left\{\begin{array}{r}
(\log t)^{\alpha-1}(1-\log s)^{\alpha-1}-(\log t-\log s)^{\alpha-1}, \\
1 \leq s \leq t \leq e, \\
(\log t)^{\alpha-1}(1-\log s)^{\alpha-1}, \\
1 \leq t \leq s \leq e .
\end{array}\right.
\end{array}
$$

Proof. Using Lemma 2, we have

$$
\begin{aligned}
u(t)= & c_{1}(\log t)^{\alpha-1}+c_{2}(\log t)^{\alpha-2}+\cdots+c_{n}(\log t)^{\alpha-n} \\
& -\frac{1}{\Gamma(\alpha)} \int_{1}^{t}(\log t-\log s)^{\alpha-1} f(s) \frac{d s}{s}
\end{aligned}
$$

where $c_{i} \in \mathbb{R}, i=1,2, \ldots, n$. By $u^{(j)}(1)=0, j=0,1, \ldots$, $n-2$, we have $c_{i}=0, i=2,3, \ldots, n$. Hence,

$$
u(t)=c_{1}(\log t)^{\alpha-1}-\frac{1}{\Gamma(\alpha)} \int_{1}^{t}(\log t-\log s)^{\alpha-1} f(s) \frac{d s}{s} .
$$

Then, we know $u(e)=c_{1}-(1 / \Gamma(\alpha)) \int_{1}^{e}(1-\log s)^{\alpha-1} f(s)$ $(d s / s)$. Using the condition $u(e)=\int_{1}^{e} h(t) u(t)(d t / t)$, we have

$$
\begin{aligned}
c_{1} & -\frac{1}{\Gamma(\alpha)} \int_{1}^{e}(1-\log s)^{\alpha-1} f(s) \frac{d s}{s} \\
= & c_{1} \int_{1}^{e} h(t)(\log t)^{\alpha-1} \frac{d t}{t} \\
& \quad-\frac{1}{\Gamma(\alpha)} \int_{1}^{e} h(t) \int_{1}^{t}(\log t-\log s)^{\alpha-1} f(s) \frac{d s}{s} \frac{d t}{t} .
\end{aligned}
$$


Then, (H0) implies that

$$
\begin{aligned}
c_{1}= & \frac{1}{\Gamma(\alpha)\left(1-\int_{1}^{e} h(t)(\log t)^{\alpha-1}(d t / t)\right)} \int_{1}^{e}(1-\log s)^{\alpha-1} f(s) \frac{d s}{s} \\
& -\frac{1}{\Gamma(\alpha)\left(1-\int_{1}^{e} h(t)(\log t)^{\alpha-1}(d t / t)\right)} \int_{1}^{e} h(t) \int_{1}^{t}(\log t-\log s)^{\alpha-1} f(s) \frac{d s}{s} \frac{d t}{t} \\
= & \frac{1}{\Gamma(\alpha)\left(1-\int_{1}^{e} h(t)(\log t)^{\alpha-1}(d t / t)\right)} \int_{1}^{e}(1-\log s)^{\alpha-1} f(s) \frac{d s}{s} \\
& -\frac{1}{\Gamma(\alpha)\left(1-\int_{1}^{e} h(t)(\log t)^{\alpha-1}(d t / t)\right)} \int_{1}^{e}(1-\log s)^{\alpha-1} f(s) \frac{d s}{s} .
\end{aligned}
$$

As a result, we have

$$
\begin{aligned}
& u(t)=\frac{(\log t)^{\alpha-1}}{\Gamma(\alpha)\left(1-\int_{1}^{e} h(t)(\log t)^{\alpha-1}(d t / t)\right)} \int_{1}^{e}(1-\log s)^{\alpha-1} f(s) \frac{d s}{s} \\
& -\frac{(\log t)^{\alpha-1}}{\Gamma(\alpha)\left(1-\int_{1}^{e} h(t)(\log t)^{\alpha-1}(d t / t)\right)} \int_{1}^{e} h(t) \int_{s}^{e}(\log t-\log s)^{\alpha-1} f(s) \frac{d t}{t} \frac{d s}{s}-\frac{1}{\Gamma(\alpha)} \int_{1}^{t}(\log t-\log s)^{\alpha-1} f(s) \frac{d s}{s} \\
& =\frac{(\log t)^{\alpha-1}}{\Gamma(\alpha)\left(1-\int_{1}^{e} h(t)(\log t)^{\alpha-1}(d t / t)\right)} \int_{1}^{e}(1-\log s)^{\alpha-1} f(s) \frac{d s}{s}+\frac{1}{\Gamma(\alpha)} \int_{1}^{e}(\log t)^{\alpha-1}(1-\log s)^{\alpha-1} f(s) \frac{d s}{s} \\
& -\frac{(\log t)^{\alpha-1}}{\Gamma(\alpha)\left(1-\int_{1}^{e} h(t)(\log t)^{\alpha-1}(d t / t)\right)} \int_{1}^{e} h(t) \int_{1}^{e}(\log t-\log s)^{\alpha-1} f(s) \frac{d t}{t} \frac{d s}{s}-\frac{1}{\Gamma(\alpha)} \int_{1}^{t}(\log t-\log s)^{\alpha-1} f(s) \frac{d s}{s} \\
& -\frac{1}{\Gamma(\alpha)} \int_{1}^{e}(\log t)^{\alpha-1}(1-\log s)^{\alpha-1} f(s) \frac{d s}{s} \\
& =\int_{1}^{e} G_{1}(t, s) f(s) \frac{d s}{s}+\frac{\int_{1}^{e} h(t)(\log t)^{\alpha-1}(d t / t)}{\Gamma(\alpha)\left(1-\int_{1}^{e} h(t)(\log t)^{\alpha-1}(d t / t)\right)} \int_{1}^{e}(\log t)^{\alpha-1}(1-\log s)^{\alpha-1} f(s) \frac{d s}{s} \\
& -\frac{(\log t)^{\alpha-1}}{\Gamma(\alpha)\left(1-\int_{1}^{e} h(t)(\log t)^{\alpha-1}(d t / t)\right)} \int_{1}^{e} h(t) \int_{s}^{e}(\log t-\log s)^{\alpha-1} f(s) \frac{d t}{t} \frac{d s}{s} \\
& =\int_{1}^{e} G_{1}(t, s) f(s) \frac{d s}{s}+\frac{(\log t)^{\alpha-1}\left[\int_{1}^{e} h(t)(\log t)^{\alpha-1}(d t / t) \int_{1}^{e}(1-\log s)^{\alpha-1} f(s)(d s / s)-\int_{1}^{e} h(t) \int_{s}^{e}(\log t-\log s)^{\alpha-1} f(s)(d t / t)(d s / s)\right]}{\Gamma(\alpha)\left(1-\int_{1}^{e} h(t)(\log t)^{\alpha-1}(d t / t)\right)} \\
& =\int_{1}^{e} G_{1}(t, s) f(s) \frac{d s}{s}+\frac{(\log t)^{\alpha-1}}{1-\int_{1}^{e} h(t)(\log t)^{\alpha-1}(d t / t)} \int_{1}^{e} \int_{1}^{e} h(t) G_{1}(t, s) \frac{d t}{t} f(s) \frac{d s}{s} \\
& =\int_{1}^{e} G(t, s) f(s) \frac{d s}{s} \text {. }
\end{aligned}
$$

This completes the proof.

In what follows, we study some useful inequalities for Green's functions in (11). We first provide a result in [1].

Let $h(t) \in C[0,1]$, and then the Riemann-Liouville boundary-value problem

$$
\begin{cases}D_{0+}^{\alpha} u(t)+h(t)=0, & 0<t<1,2 \leq n-1<\alpha \leq n, \\ u^{(j)}(0)=u(1)=0, & 0 \leq j \leq n-2,\end{cases}
$$

has a unique solution $u(t)=\int_{0}^{1} H(t, s) h(s) d s$, where 


$$
H(t, s)=\frac{1}{\Gamma(\alpha)} \begin{cases}t^{\alpha-1}(1-s)^{\alpha-1}-(t-s)^{\alpha-1}, & 0 \leq s \leq t \leq 1 \\ t^{\alpha-1}(1-s)^{\alpha-1}, & 0 \leq t \leq s \leq 1\end{cases}
$$

Moreover, Green's function $H$ satisfies the inequalities:

$\Gamma(\alpha) k(t) q(s) \leq H(t, s) \leq(\alpha-1) q(s), \quad$ for $t, s \in[0,1]$,

where $k(t)=\left(t^{\alpha-1}(1-t) / \Gamma(\alpha)\right)$ and $q(s)=\left(s(1-s)^{\alpha-1} / \Gamma(\alpha)\right)$.

Comparing $G_{1}$ with $H$, using $\log t$ and $\log s$ to replace $t$ and $s$, from (11) and (19), we obtain the function $G_{1}$ satisfies the inequalities:

$$
\begin{gathered}
\frac{(\log t)^{\alpha-1}(1-\log t)(\log s)(1-\log s)^{\alpha-1}}{\Gamma(\alpha)} \leq G_{1}(t, s) \\
\leq \frac{(\alpha-1)(\log s)(1-\log s)^{\alpha-1}}{\Gamma(\alpha)}, \quad t, s \in[1, e] .
\end{gathered}
$$

This, for all $t, s \in[1, e]$, implies that

$$
\begin{aligned}
& \frac{(\log t)^{\alpha-1}}{1-\int_{1}^{e} h(t)(\log t)^{\alpha-1}(d t / t)} \int_{1}^{e} h(t) G_{1}(t, s) \frac{d t}{t} \\
& \leq \frac{(\alpha-1) \int_{1}^{e} h(t)(d t / t)}{\Gamma(\alpha)\left(1-\int_{1}^{e} h(t)(\log t)^{\alpha-1}(d t / t)\right)}(\log s)(1-\log s)^{\alpha-1},
\end{aligned}
$$

and

$$
\begin{gathered}
\frac{(\log t)^{\alpha-1}}{1-\int_{1}^{e} h(t)(\log t)^{\alpha-1}(d t / t)} \int_{1}^{e} h(t) G_{1}(t, s) \frac{d t}{t} \\
\geq \frac{(\log t)^{\alpha-1} \int_{1}^{e} h(t)(\log t)^{\alpha-1}(1-\log t)(d t / t)}{\Gamma(\alpha)\left(1-\int_{1}^{e} h(t)(\log t)^{\alpha-1}(d t / t)\right)} \\
\cdot(\log s)(1-\log s)^{\alpha-1} .
\end{gathered}
$$

Lemma 4. Let $\phi(t)=(\log t)(1-\log t)^{\alpha-1}$, where $t \in[1, e]$. Then there exist

$$
\begin{aligned}
& \kappa_{1}=\frac{\alpha^{2} \Gamma(\alpha)}{\Gamma(2 \alpha+2)}+\frac{\Gamma(\alpha)}{2 \Gamma(2 \alpha)} \frac{\int_{1}^{e} h(t)(\log t)^{\alpha-1}(1-\log t)(d t / t)}{1-\int_{1}^{e} h(t)(\log t)^{\alpha-1}(d t / t)}, \\
& \kappa_{2}=\frac{\alpha-1}{\Gamma(\alpha+2)}\left[1+\frac{\int_{1}^{e} h(t)(d t / t)}{1-\int_{1}^{e} h(t)(\log t)^{\alpha-1}(d t / t)}\right]
\end{aligned}
$$

such that

$$
\kappa_{1} \phi(s) \leq \int_{1}^{e} G(t, s) \phi(t) \frac{d t}{t} \leq \kappa_{2} \phi(s), \quad \text { for } s \in[1, e] .
$$

Proof. Using (20)-(22), for all $s \in[1, e]$, we have

$$
\begin{aligned}
\int_{1}^{e} G(t, s) \phi(t) \frac{d t}{t} \leq & \int_{1}^{e} \frac{(\alpha-1) \phi(s)}{\Gamma(\alpha)} \phi(t) \frac{d t}{t} \\
& +\int_{1}^{e} \frac{(\alpha-1) \int_{1}^{e} h(t)(d t / t)}{\Gamma(\alpha)\left(1-\int_{1}^{e} h(t)(\log t)^{\alpha-1}(d t / t)\right)} \\
& \cdot \phi(s) \phi(t) \frac{d t}{t}=\kappa_{2} \phi(s), \\
\int_{1}^{e} G(t, s) \phi(t) \frac{d t}{t} \geq & \int_{1}^{e} \frac{(\log t)^{\alpha-1}(1-\log t) \phi(s)}{\Gamma(\alpha)} \phi(t) \frac{d t}{t} \\
& +\int_{1}^{e} \frac{(\log t)^{\alpha-1} \int_{1}^{e} h(t)(\log t)^{\alpha-1}(1-\log t)(d t / t)}{\Gamma(\alpha)\left(1-\int_{1}^{e} h(t)(\log t)^{\alpha-1}(d t / t)\right)} \\
& \cdot \phi(s) \phi(t) \frac{d t}{t}=\kappa_{1} \phi(s) .
\end{aligned}
$$

This completes the proof.

From Lemma 3, we know (1) is equivalent to the following Hammerstein-type integral equations:

$$
\left(\begin{array}{l}
u(t) \\
v(t)
\end{array}\right)=\left(\begin{array}{l}
\int_{1}^{e} G(t, s) f_{1}(s, u(s), v(s)) \frac{d s}{s} \\
\int_{1}^{e} G(t, s) f_{2}(s, u(s), v(s)) \frac{d s}{s}
\end{array}\right) .
$$

Let $E:=C[1, e],|u|:=\max _{t \in[1, e]}|u(t)|$, and $P:=\{u \in$ $E: u(t) \geq 0, \forall t \in[1, e]\}$. Then $(E,\|\cdot\|)$ becomes a real Banach space and $P$ a cone on $E$. Moreover, $E \times E$ is a Banach space with the norm $(x, y)=\|x\|+\|y\|$, and $P \times P$ is a cone on $E \times E$. Therefore, we define operators $A_{i}(i=1,2)$ and $A$ as follows:

$$
\begin{aligned}
& A_{1}(u, v)(t)=\int_{1}^{e} G(t, s) f_{1}(s, u(s), v(s)) \frac{d s}{s} \\
& A_{2}(u, v)(t)=\int_{1}^{e} G(t, s) f_{2}(s, u(s), v(s)) \frac{d s}{s} \\
& A(u, v)(t)=\left(A_{1}, A_{2}\right)(u, v)(t), \quad \text { for } u, v \in P, t \in[1, e] .
\end{aligned}
$$

Note that $G$ and $f_{i}(i=1,2)$ are nonnegative continuous functions, so the operators $A_{i}: P \times P \longrightarrow P(i=1,2)$ and $A$ : $P \times P \longrightarrow P \times P$ are three completely continuous operators. Moreover, if $(u, v) \in(P \times P) \backslash\{0\}$ is a fixed point of $A$, then $(u, v)$ is a positive solution for $(1)$. Therefore, in what follows, we turn to study the existence of fixed points of the operator $A$.

Lemma 5. Let $p$ be a continuous concave function. Then, if $\varphi$ is an integrable function on $[0,1]$, we have

$$
p\left(\int_{0}^{1} \varphi(t) d t\right) \geq \int_{0}^{1} p(\varphi(t)) d t
$$


Proof. Let $0=t_{0}<t_{1}<t_{2}<\cdots<t_{n-1}<t_{n}=1$, for all $n \in \mathbb{N}_{+}$, and $\Delta t_{i}=t_{i}-t_{i-1}, d=\max \left\{\Delta t_{i}\right\}, i=1,2, \ldots, n$. Then, note that $\sum_{i=1}^{n} \Delta t_{i}=1$, for all $\xi_{i} \in\left[t_{i-1}, t_{i}\right]$, where $i=1,2, \ldots, n$, we have

$$
\begin{aligned}
p\left(\int_{0}^{1} \varphi(t) d t\right)= & p\left(\lim _{d \longrightarrow 0} \sum_{i=1}^{n} \varphi\left(\xi_{i}\right) \Delta t_{i}\right)=\lim _{d \longrightarrow 0} p\left(\sum_{i=1}^{n} \varphi\left(\xi_{i}\right) \Delta t_{i}\right) \\
& \geq \lim _{d \longrightarrow 0} \sum_{i=1}^{n} p\left(\varphi\left(\xi_{i}\right)\right) \Delta t_{i}=\int_{0}^{1} p(\varphi(t)) d t .
\end{aligned}
$$

This completes the proof.

Remark 1. If $p$ is a continuous convex function in Lemma 5 , then (28) can be changed into the inverse inequality:

$$
p\left(\int_{0}^{1} \varphi(t) d t\right) \leq \int_{0}^{1} p(\varphi(t)) d t
$$

Lemma 6 (see [34]). Let $E$ be a real Banach space and $P$ a cone on E. Suppose that $\Omega \subset E$ is a bounded open set and that $A: \bar{\Omega} \cap P \longrightarrow P$ is a continuous compact operator. If there exists a $\omega_{0} \in P \backslash\{0\}$ such that

$$
\omega-A \omega \neq \lambda \omega_{0}, \quad \forall \lambda \geq 0, \omega \in \partial \Omega \cap P,
$$

then $i(A, \Omega \cap P, P)=0$, where $i$ denotes the fixed-point index on $P$.
Lemma 7 (see [34]). Let $E$ be a real Banach space and $P$ a cone on $E$. Suppose that $\Omega \subset E$ is a bounded open set with $0 \in \Omega$ and that $A: \bar{\Omega} \cap P \longrightarrow P$ is a continuous compact operator. If

$$
\omega-\lambda A \omega \neq 0, \quad \forall \lambda \in[0,1], \omega \in \partial \Omega \cap P,
$$

then $i(A, \Omega \cap P, P)=1$.

\section{Main Results}

Lemma 8. Let $P_{0}=\left\{u \in P: \int_{1}^{e} u(t) \phi(t)(d t / t) \geq \omega_{0}\|u\|\right\}$. Then $B u \in P_{0}$, where

$$
(B u)(t)=\int_{1}^{e} G(t, s) u(s) \frac{d s}{s}, \quad u \in P,
$$

where

$$
\begin{aligned}
\omega_{0}= & \frac{\alpha^{2} \Gamma^{2}(\alpha)}{(\alpha-1) \Gamma(2 \alpha+2)}\left[1+\frac{\int_{1}^{e} h(t)(\log t)^{\alpha-1}(1-\log t)(d t / t)}{1-\int_{1}^{e} h(t)(\log t)^{\alpha-1}(d t / t)}\right] \\
& \cdot\left[1+\frac{\int_{1}^{e} h(t)(d t / t)}{1-\int_{1}^{e} h(t)(\log t)^{\alpha-1}(d t / t)}\right]^{-1} .
\end{aligned}
$$

Proof. From the definition of $G$, for all $t, \tau, s \in[1, e]$, we have

$$
\begin{aligned}
G(t, s) \geq & \frac{(\log t)^{\alpha-1}(1-\log t) \phi(s)}{\Gamma(\alpha)}+\frac{(\log t)^{\alpha-1} \int_{1}^{e} h(t)(\log t)^{\alpha-1}(1-\log t)(d t / t)}{\Gamma(\alpha)\left(1-\int_{1}^{e} h(t)(\log t)^{\alpha-1}(d t / t)\right)} \phi(s) \\
\geq & \frac{(\log t)^{\alpha-1}(1-\log t)}{\Gamma(\alpha)}\left[1+\frac{\int_{1}^{e} h(t)(\log t)^{\alpha-1}(1-\log t)(d t / t)}{1-\int_{1}^{e} h(t)(\log t)^{\alpha-1}(d t / t)}\right] \phi(s) \\
= & \frac{(\log t)^{\alpha-1}(1-\log t)}{\Gamma(\alpha)}\left[1+\frac{\int_{1}^{e} h(t)(\log t)^{\alpha-1}(1-\log t)(d t / t)}{1-\int_{1}^{e} h(t)(\log t)^{\alpha-1}(d t / t)}\right] \\
& \cdot \frac{\alpha-1}{\Gamma(\alpha)}\left[1+\frac{\int_{1}^{e} h(t)(d t / t)}{1-\int_{1}^{e} h(t)(\log t)^{\alpha-1}(d t / t)}\right] \phi(s) \cdot \frac{\Gamma(\alpha)}{\alpha-1}\left[1+\frac{\int_{1}^{e} h(t)(d t / t)}{1-\int_{1}^{e} h(t)(\log t)^{\alpha-1}(d t / t)}\right]^{-1} \\
\geq & \frac{(\log t)^{\alpha-1}(1-\log t)}{\alpha-1}\left[1+\frac{\int_{1}^{e} h(t)(\log t)^{\alpha-1}(1-\log t)(d t / t)}{1-\int_{1}^{e} h(t)(\log t)^{\alpha-1}(d t / t)}\right]\left[1+\frac{\int_{1}^{e} h(t)(d t / t)}{1-\int_{1}^{e} h(t)(\log t)^{\alpha-1}(d t / t)}\right]^{-1} G(\tau, s) .
\end{aligned}
$$


Then if $u \in P$, we have

$$
\begin{aligned}
\int_{1}^{e}(B u)(t) \phi(t) \frac{d t}{t} & =\int_{1}^{e} \phi(t) \int_{1}^{e} G(t, s) u(s) \frac{d s}{s} \frac{d t}{t} \\
\geq & \int_{1}^{e} \phi(t) \int_{1}^{e} \frac{(\log t)^{\alpha-1}(1-\log t)}{\alpha-1}\left[1+\frac{\int_{1}^{e} h(t)(\log t)^{\alpha-1}(1-\log t)(d t / t)}{1-\int_{1}^{e} h(t)(\log t)^{\alpha-1}(d t / t)}\right] \\
& \cdot\left[1+\frac{\int_{1}^{e} h(t)(d t / t)}{1-\int_{1}^{e} h(t)(\log t)^{\alpha-1}(d t / t)}\right]^{-1} G(\tau, s) u(s) \frac{d s}{s} \frac{d t}{t} \\
= & \frac{\alpha^{2} \Gamma^{2}(\alpha)}{(\alpha-1) \Gamma(2 \alpha+2)}\left[1+\frac{\int_{1}^{e} h(t)(\log t)^{\alpha-1}(1-\log t)(d t / t)}{1-\int_{1}^{e} h(t)(\log t)^{\alpha-1}(d t / t)}\right]\left[1+\frac{\int_{1}^{e} h(t)(d t / t)}{1-\int_{1}^{e} h(t)(\log t)^{\alpha-1}(d t / t)}\right]^{-1}(B u)(\tau)
\end{aligned}
$$

Note that the arbitrariness of $\tau \in[0,1]$, we have

$$
\int_{1}^{e}(B u)(t) \phi(t) \frac{d t}{t} \geq \omega_{0}\|B u\|
$$

This completes the proof.

Let $\quad \mathscr{K}=(\alpha-1 / \Gamma(\alpha))\left[1+\left(\int_{1}^{e} h(t)(d t / t) / 1-\int_{1}^{e} h(t)\right.\right.$ $\left.\left.(\log t)^{\alpha-1}(d t / t)\right)\right]$. Then, $\max _{t, s \in[1, e]} G(t, s) \leq \mathscr{K}$. Now, we list our assumptions for $f_{i}(i=1,2)$ :

(H1) $f_{i} \in C\left([1, e] \times \mathbb{R}^{+} \times \mathbb{R}^{+}, \mathbb{R}^{+}\right), i=1,2$.

(H2) There exist $p_{1}, q_{1} \in C\left(\mathbb{R}^{+}, \mathbb{R}^{+}\right)$and $c_{1}>0$ such that

(i) $p_{1}$ is a strictly increasing concave function on $\mathbb{R}^{+}$ and $\lim _{z \longrightarrow+\infty} p_{1}(z)=+\infty$

(ii) $\left(\begin{array}{l}f_{1}(t, u, v) \\ f_{2}(t, u, v)\end{array}\right) \geq\left(\begin{array}{c}p_{1}(v)-c_{1} \\ q_{1}(u)-c_{1}\end{array}\right), \forall(t, u, v) \in[1, e] \times$ $\mathbb{R}^{+} \times \mathbb{R}^{+}$

(iii) $\exists \gamma_{1} \in\left(\kappa_{1}^{-2},+\infty\right)$ such that $p_{1}\left(\mathscr{K} q_{1}(z)\right) \geq \gamma_{1} \mathscr{K} z-$ $c_{1}, \forall z \in \mathbb{R}^{+}$

(H3) There exist $p_{2}, q_{2} \in C\left(\mathbb{R}^{+}, \mathbb{R}^{+}\right)$and $r_{1}>0$ such that

(i) $p_{2}$ is a strictly increasing convex function on $\mathbb{R}^{+}$ and $p_{2}(0)=0$

(ii) $\left(\begin{array}{l}f_{1}(t, u, v) \\ f_{2}(t, u, v)\end{array}\right) \leq\left(\begin{array}{l}p_{2}(v) \\ q_{2}(u)\end{array}\right), \forall(t, u, v) \in[1, e] \times\left[0, r_{1}\right] \times$ $\left[0, r_{1}\right]$

(iii) $\exists \gamma_{2} \in\left(0, \kappa_{2}^{-2}\right)$ such that $p_{2}\left(\mathscr{K} q_{2}(z)\right) \leq \gamma_{2} \mathscr{K} z$, $\forall z \in\left[0, r_{1}\right]$

(H4) There exist $p_{3}, q_{3} \in C\left(\mathbb{R}^{+}, \mathbb{R}^{+}\right)$and $r_{2}>0$ such that

(i) $p_{3}$ is a strictly increasing concave function on $\mathbb{R}^{+}$

(ii) $\left(\begin{array}{l}f_{1}(t, u, v) \\ f_{2}(t, u, v)\end{array}\right) \geq\left(\begin{array}{l}p_{3}(v) \\ q_{3}(u)\end{array}\right), \forall(t, u, v) \in[1, e] \times\left[0, r_{2}\right] \times$ $\left[0, r_{2}\right]$

(iii) $\exists \gamma_{3} \in\left(\kappa_{1}^{-2},+\infty\right)$ such that $p_{3}\left(\mathscr{K} q_{3}(z)\right) \geq \gamma_{3} \mathscr{K} z$, $\forall z \in\left[0, r_{2}\right]$

(H5) There exist $a_{11}, b_{11}, a_{12}, b_{12} \geq 0$ and $l_{1}, l_{2}>0$ such that

$$
\begin{aligned}
& a_{11} \kappa_{2}<1, b_{12} \kappa_{2}<1, \operatorname{det}\left(\begin{array}{cc}
1-a_{11} \kappa_{2} & -a_{12} \kappa_{2} \\
-b_{11} \kappa_{2} & 1-b_{12} \kappa_{2}
\end{array}\right)>0, \\
& \left(\begin{array}{l}
f_{1}(t, u, v) \\
f_{2}(t, u, v)
\end{array}\right) \leq\left(\begin{array}{l}
a_{11} u+a_{12} v+l_{1} \\
b_{11} u+b_{12} v+l_{2}
\end{array}\right), \quad \forall(t, u, v) \in[1, e] \times \mathbb{R}^{+} \times \mathbb{R}^{+} .
\end{aligned}
$$

Define $B_{\rho}=\{z \in E:\|z\|<\rho\}$ for $\rho>0$. We adopt the convention in the sequel that $c_{1}, c_{2}, \ldots$ stand for different positive constants.

Theorem 1. Suppose that (H1)-(H3) hold. Then, (1) has at least one positive solution.

Proof. Let $M_{1}=\left\{(u, v) \in P \times P:(u, v)=A(u, v)+\mu\left(u^{*}, v^{*}\right)\right.$, $\mu \geq 0\}$, where $u^{*}, v^{*} \in P_{0}$ are two given elements. Then, we claim that $M_{1}$ is a bounded set in $P \times P$. We define operators $\mathbf{f}_{i}: P \times P \longrightarrow P(i=1,2)$ as follows:

$\mathbf{f}_{i}(u, v)(t)=f_{i}(t, u(t), v(t)), \quad$ for $u, v \in P, t \in[1, e], i=1,2$.

Now, if there exists $(u, v) \in M_{1}$, then we have $u=$ $A_{1}(u, v)+\mu u^{*}=B \mathbf{f}_{1}(u, v)+\mu u^{*}$ and $v=A_{2}(u, v)+\mu v^{*}=$ $B \mathbf{f}_{2}(u, v)+\mu v^{*}$. From Lemma 8 , we have

$$
u, v \in P_{0}
$$

Moreover, together with (H2) (ii), we can obtain that

$$
\begin{aligned}
u(t) & \geq A_{1}(u, v)(t) \geq \int_{1}^{e} G(t, s)\left(p_{1}(v(s))-c_{1}\right) \frac{d s}{s}, \\
v(t) & \geq A_{2}(u, v)(t) \geq \int_{1}^{e} G(t, s)\left(q_{1}(u(s))-c_{1}\right) \frac{d s}{s}, \\
& \geq \int_{1}^{e} G(t, s) q_{1}(u(s)) \frac{d s}{s}-c_{2} .
\end{aligned}
$$

Using (H2) (i) and (iii), we have 


$$
\begin{aligned}
p_{1}(v(t)) & \geq p_{1}\left(v(t)+c_{2}\right)-p_{1}\left(c_{2}\right) \\
& \geq p_{1}\left(\int_{1}^{e} G(t, s) q_{1}(u(s)) \frac{d s}{s}\right)-p_{1}\left(c_{2}\right) \\
& =p_{1}\left(\int_{0}^{1} G\left(t, e^{x}\right) q_{1}\left(u\left(e^{x}\right)\right) d x\right)-p_{1}\left(c_{2}\right) \\
& \geq \int_{0}^{1} p_{1}\left(G\left(t, e^{x}\right) q_{1}\left(u\left(e^{x}\right)\right)\right) d x-p_{1}\left(c_{2}\right) \\
& =\int_{0}^{1} p_{1}\left(\frac{G\left(t, e^{x}\right)}{\mathscr{K}} \mathscr{K} q_{1}\left(u\left(e^{x}\right)\right)+\left(1-\frac{G\left(t, e^{x}\right)}{\mathscr{K}}\right) \cdot 0\right) d x-p_{1}\left(c_{2}\right) \\
& \geq \int_{0}^{1} \frac{G\left(t, e^{x}\right)}{\mathscr{K}} p_{1}\left(\mathscr{K} q_{1}\left(u\left(e^{x}\right)\right)\right) d x-p_{1}\left(c_{2}\right) \\
& \geq \int_{0}^{1} \frac{G\left(t, e^{x}\right)}{\mathscr{K}}\left(\gamma_{1} \mathscr{K} u\left(e^{x}\right)-c_{1}\right) d x-p_{1}\left(c_{2}\right) \\
& \geq \gamma_{1} \int_{1}^{e} G(t, s) u(s) \frac{d s}{s}-c_{3} .
\end{aligned}
$$

Therefore, we have

$$
\begin{aligned}
u(t) & \geq \int_{1}^{e} G(t, s)\left(\gamma_{1} \int_{1}^{e} G(s, \tau) u(\tau) \frac{d \tau}{\tau}-c_{3}\right) \frac{d s}{s}-c_{2} \\
& \geq \gamma_{1} \int_{1}^{e} \int_{1}^{e} G(t, s) G(s, \tau) u(\tau) \frac{d s}{s} \frac{d \tau}{\tau}-c_{4} .
\end{aligned}
$$

Recall that $\phi(t)=(\log t)(1-\log t)^{\alpha-1}$, where $t \in[1, e]$. Therefore, we multiply both sides of the above by $\phi(t)$, integrate over $[1, e]$, and use Lemma 4 to obtain

$$
\begin{aligned}
\int_{1}^{e} u(t) \phi(t) \frac{d t}{t} \geq & \gamma_{1} \int_{1}^{e} \phi(t) \int_{1}^{e} \int_{1}^{e} G(t, s) G(s, \tau) u(\tau) \frac{d s}{s} \frac{d \tau}{\tau} \frac{d t}{t} \\
& -c_{4} \int_{1}^{e} \phi(t) \frac{d t}{t} \geq \gamma_{1} \kappa_{1}^{2} \int_{1}^{e} u(t) \phi(t) \frac{d t}{t}-\frac{c_{4} \Gamma(\alpha)}{\Gamma(\alpha+2)} .
\end{aligned}
$$

Solving this inequality, from (40), we have

$$
\|u\| \leq \omega_{0}^{-1} \int_{1}^{e} u(t) \phi(t) \frac{d t}{t} \leq \frac{\omega_{0}^{-1} c_{4} \Gamma(\alpha)}{\left(\gamma_{1} \kappa_{1}^{2}-1\right) \Gamma(\alpha+2)} .
$$

On the other hand, we estimate the norm of $v$. Multiplying both sides of the first inequality of (41) by $\phi(t)$, integrating over $[1, e]$, and using Lemma 4 , we obtain

$$
\int_{1}^{e} u(t) \phi(t) \frac{d t}{t} \geq \kappa_{1} \int_{1}^{e} \phi(t)\left(p_{1}(v(t))-c_{1}\right) \frac{d t}{t} .
$$

This implies that

$$
\int_{1}^{e} \phi(t) p_{1}(v(t)) \frac{d t}{t} \leq \frac{\kappa_{1}^{-1} c_{4} \Gamma(\alpha)}{\left(\gamma_{1} \kappa_{1}^{2}-1\right) \Gamma(\alpha+2)}+\frac{c_{1} \Gamma(\alpha)}{\Gamma(\alpha+2)} .
$$

Without loss of generality, we may assume $v(t) \not \equiv 0$, then $v>0$. Note that $v \in P_{0}$, we have $\|v\| \leq \frac{1}{\omega_{0}} \int_{1}^{e} v(t) \phi(t) \frac{d t}{t}=\frac{\|v\|}{\omega_{0} p_{1}(\|v\|)} \int_{0}^{1} \frac{v\left(e^{x}\right)}{\|v\|} p_{1}(\|v\|) \phi\left(e^{x}\right) d x$

$$
\leq \frac{\|v\|}{\omega_{0} p_{1}(\|v\|)} \int_{0}^{1} p_{1}\left(v\left(e^{x}\right)\right) \phi\left(e^{x}\right) d x
$$

$p_{1}(\|v\|) \leq \frac{1}{\omega_{0}} \int_{0}^{1} p_{1}\left(v\left(e^{x}\right)\right) \phi\left(e^{x}\right) d x=\frac{1}{\omega_{0}} \int_{1}^{e} p_{1}(v(t)) \phi(t) \frac{d t}{t}$

$$
\leq \frac{1}{\omega_{0}}\left[\frac{\kappa_{1}^{-1} c_{4} \Gamma(\alpha)}{\left(\gamma_{1} \kappa_{1}^{2}-1\right) \Gamma(\alpha+2)}+\frac{c_{1} \Gamma(\alpha)}{\Gamma(\alpha+2)}\right] .
$$

Combining (H2) (i) $\left(\lim _{z \longrightarrow+\infty} p_{1}(z)=+\infty\right)$, there exists $\mathcal{N}_{1}$ such that $\|v\| \leq \mathcal{N}_{1}$.

Up to now, we have proved the boundedness of $M_{1}$. Taking $R_{1}>\mathcal{N}_{1}+\left(\omega_{0}^{-1} c_{4} \Gamma(\alpha) /\left(\gamma_{1} \kappa_{1}^{2}-1\right) \Gamma(\alpha+2)\right) \quad$ and $R_{1}>r_{1}\left(r_{1}\right.$ is defined by $\left.(\mathrm{H} 3)\right)$, we have

$(u, v) \neq A(u, v)+\mu\left(u^{*}, v^{*}\right)$, for $(u, v) \in \partial B_{R_{1}} \cap(P \times P), \mu \geq 0$.

Then, Lemma 6 enables us to obtain

$$
i\left(A, B_{R_{1}} \cap(P \times P), P \times P\right)=0 .
$$

Next, we show that

$(u, v) \neq \mu A(u, v)$, for $(u, v) \in \partial B_{r_{1}} \cap(P \times P), \mu \in[0,1]$.

If this claim is not true, then there exist $(u, v) \in \partial B_{r_{1}} \cap$ $(P \times P), \mu \in[0,1]$ such that

$$
(u, v)=\mu A(u, v) .
$$

Combining (H3) (ii), we obtain

$$
\begin{aligned}
& u(t) \leq A_{1}(u, v)(t) \leq \int_{1}^{e} G(t, s) p_{2}(v(s)) \frac{d s}{s}, \\
& v(t) \leq A_{2}(u, v)(t) \leq \int_{1}^{e} G(t, s) q_{2}(u(s)) \frac{d s}{s} .
\end{aligned}
$$

From (H3) (i) and (iii), we have

$$
\begin{aligned}
p_{2}(v(t)) & \leq p_{2}\left(\int_{1}^{e} G(t, s) q_{2}(u(s)) \frac{d s}{s}\right) \\
& \leq \int_{0}^{1} p_{2}\left(G\left(t, e^{x}\right) q_{2}\left(u\left(e^{x}\right)\right)\right) d x \\
& =\int_{0}^{1} p_{2}\left(\frac{G\left(t, e^{x}\right)}{\mathscr{K}} \mathscr{K} q_{2}\left(u\left(e^{x}\right)\right)+\left(1-\frac{G\left(t, e^{x}\right)}{\mathscr{K}}\right) \cdot 0\right) d x \\
& \leq \int_{0}^{1} \frac{G\left(t, e^{x}\right)}{\mathscr{K}} p_{2}\left(\mathscr{K} q_{2}\left(u\left(e^{x}\right)\right)\right) d x \\
& \leq \gamma_{2} \int_{1}^{e} G(t, s) u(s) \frac{d s}{s} .
\end{aligned}
$$


Consequently, we have

$$
u(t) \leq \gamma_{2} \int_{1}^{e} \int_{1}^{e} G(t, s) G(s, \tau) u(\tau) \frac{d \tau}{\tau} \frac{d s}{s} .
$$

Multiplying both sides of the above by $\phi(t)$, integrating over $[1, e]$, and using Lemma 4 , we obtain

$$
\int_{1}^{e} u(t) \phi(t) \frac{d t}{t} \leq \gamma_{2} \kappa_{2}^{2} \int_{1}^{e} u(t) \phi(t) \frac{d t}{t} .
$$

Note that $\gamma_{2} \in\left(0, \kappa_{2}^{-2}\right)$, we have $\int_{1}^{e} u(t) \phi(t)(d t / t)=0$ and $u(t) \equiv 0$ for $t \in[1, e]$. Moreover, using (54), we have $p_{2}(v(t)) \equiv 0$ for $t \in[1, e]$. From (H3) (i), we have $v(t) \equiv 0$ for $t \in[1, e]$. Therefore, this contradicts to $(u, v) \in \partial B_{r_{1}} \cap$ $(P \times P), r_{1}>0$. This also implies that (51) holds. Then, Lemma 7 enables us to obtain

$$
i\left(A, B_{r_{1}} \cap(P \times P), P \times P\right)=1 .
$$

From (50) and (57), we have

$$
\begin{aligned}
& i\left(A,\left(B_{R_{1}} \backslash \bar{B}_{r_{1}}\right) \cap(P \times P), P \times P\right)=i\left(A, B_{R_{1}} \cap(P \times P), P \times P\right) \\
& \quad-i\left(A, B_{r_{1}} \cap(P \times P), P \times P\right)=0-1=-1 .
\end{aligned}
$$

Therefore, the operator $A$ has at least one fixed point on $\left(B_{R_{1}} \backslash \bar{B}_{r_{1}}\right) \cap(P \times P)$. Equivalently, (1) has at least one positive solution. This completes the proof.

Theorem 2. Suppose that (H1) and (H4)-(H5) hold. Then, (1) has at least one positive solution.

Proof. For $r_{2}$ in (H4), we first show that

$$
(u, v) \neq A(u, v)+\mu\left(u^{*}, v^{*}\right), \quad \text { for }(u, v) \in \partial B_{r_{2}} \cap(P \times P), \mu \geq 0,
$$

where $u^{*}, v^{*} \in P$ are two given elements. Indeed, if this claim is false, there exist $(u, v) \in \partial B_{r_{2}} \cap(P \times P), \mu \geq 0$ such that

$$
(u, v)=A(u, v)+\mu\left(u^{*}, v^{*}\right)
$$

This, together with (H4) (ii), implies that

$$
\begin{aligned}
u(t) & \geq A_{1}(u, v)(t) \geq \int_{1}^{e} G(t, s) p_{3}(v(s)) \frac{d s}{s}, v(t) \geq A_{2}(u, v)(t) \\
& \geq \int_{1}^{e} G(t, s) q_{3}(u(s)) \frac{d s}{s} .
\end{aligned}
$$

Similar to (42), we have

$$
\begin{aligned}
p_{3}(v(t)) & \geq p_{3}\left(\int_{1}^{e} G(t, s) q_{3}(u(s)) \frac{d s}{s}\right) \\
& \geq \int_{1}^{e} \frac{G(t, s)}{\mathscr{K}} p_{3}\left(\mathscr{K} q_{3}(u(s))\right) \frac{d s}{s} .
\end{aligned}
$$

$$
\begin{aligned}
u(t) & \geq \int_{1}^{e} G(t, s) p_{3}(v(s)) \frac{d s}{s} \\
& \geq \gamma_{3} \int_{1}^{e} \int_{1}^{e} G(t, s) G(s, \tau) u(\tau) \frac{d s}{s} \frac{d \tau}{\tau} .
\end{aligned}
$$

Multiplying both sides of the above by $\phi(t)$, integrating over $[1, e]$, and using Lemma 4 , we obtain

$$
\int_{1}^{e} u(t) \phi(t) \frac{d t}{t} \geq \gamma_{3} \kappa_{1}^{2} \int_{1}^{e} u(t) \phi(t) \frac{d t}{t}
$$

where $\phi(t)=(\log t)(1-\log t)^{\alpha-1}, t \in[1, e]$. Consequently, $\gamma_{3} \kappa_{1}^{2}>1$ implies that $\int_{1}^{e} u(t) \phi(t)(d t / t)=0$ and $u(t) \equiv 0$ for $t \in[1, e]$. Note that (65), should be

$$
\int_{1}^{e} G(t, s) p_{3}(v(s)) \frac{d s}{s} \leq u(t) \equiv 0, \forall t \in[1, e] .
$$

From (H4) (i), this indicates that $p_{3}(v(s)) \equiv 0$ and $v(s) \equiv 0$ for $s \in[1, e]$. Therefore, $\|u\|=\|v\|=0$ contradicts to $(u, v) \in \partial B_{r_{2}} \cap(P \times P)$ and (59) holds. Then, Lemma 6 enables us to obtain

$$
i\left(A, B_{r_{2}} \cap(P \times P), P \times P\right)=0 .
$$

Let $M_{2}=\{(u, v) \in P \times P:(u, v)=\mu A(u, v), \mu \in[0,1]\}$. Then, we prove that $M_{2}$ is a bounded set in $P \times P$. If $(u, v) \in M_{2}$, then we have

$$
\begin{aligned}
& u=\mu A_{1}(u, v), \\
& v=\mu A_{2}(u, v), \text { for }(u, v) \in P \times P .
\end{aligned}
$$

From Lemma 8, we have

$$
u, v \in P_{0} \text {. }
$$

Moreover, by (H5), we have

$$
\left(\begin{array}{l}
u(t) \\
v(t)
\end{array}\right) \leq\left(\begin{array}{l}
\int_{1}^{e} G(t, s)\left(a_{11} u(s)+a_{12} v(s)+l_{1}\right) \frac{d s}{s} \\
\int_{1}^{e} G(t, s)\left(b_{11} u(s)+b_{12} v(s)+l_{2}\right) \frac{d s}{s}
\end{array}\right) .
$$

Multiplying both sides of the above by $\phi(t)$, integrating over $[1, e]$, and using Lemma 4 , we obtain

$$
\left(\begin{array}{l}
\int_{1}^{e} u(t) \phi(t) \frac{d t}{t} \\
\int_{1}^{e} v(t) \phi(t) \frac{d t}{t}
\end{array}\right) \leq\left(\begin{array}{l}
\kappa_{2} \int_{1}^{e} \phi(t)\left(a_{11} u(t)+a_{12} v(t)+l_{1}\right) \frac{d t}{t} \\
\kappa_{2} \int_{1}^{e} \phi(t)\left(b_{11} u(t)+b_{12} v(t)+l_{2}\right) \frac{d t}{t}
\end{array}\right) .
$$

Consequently, we have

$$
\left(\begin{array}{cc}
1-a_{11} \kappa_{2} & -a_{12} \kappa_{2} \\
-b_{11} \kappa_{2} & 1-b_{12} \kappa_{2}
\end{array}\right)\left(\begin{array}{c}
\int_{1}^{e} u(t) \phi(t) \frac{d t}{t} \\
\int_{1}^{e} v(t) \phi(t) \frac{d t}{t}
\end{array}\right) \leq\left(\begin{array}{c}
\frac{\kappa_{2} l_{1} \Gamma(\alpha)}{\Gamma(\alpha+2)} \\
\frac{\kappa_{2} l_{2} \Gamma(\alpha)}{\Gamma(\alpha+2)}
\end{array}\right) .
$$




$$
\left(\begin{array}{l}
\int_{1}^{e} u(t) \phi(t) \frac{d t}{t} \\
\int_{1}^{e} v(t) \phi(t) \frac{d t}{t}
\end{array}\right) \leq \frac{\left(\begin{array}{cc}
1-b_{12} \kappa_{2} & a_{12} \kappa_{2} \\
b_{11} \kappa_{2} & 1-a_{11} \kappa_{2}
\end{array}\right)\left(\begin{array}{l}
\kappa_{2} l_{1} \Gamma(\alpha) / \Gamma(\alpha+2) \\
\kappa_{2} l_{2} \Gamma(\alpha) / \Gamma(\alpha+2)
\end{array}\right)}{\left(1-a_{11} \kappa_{2}\right)\left(1-b_{12} \kappa_{2}\right)-a_{12} b_{11} \kappa_{2}^{2}} .
$$

This implies that

$$
\begin{aligned}
& \int_{1}^{e} u(t) \phi(t) \frac{d t}{t} \leq \frac{\left(\kappa_{2} \Gamma(\alpha) / \Gamma(\alpha+2)\right)\left[\left(1-b_{12} \kappa_{2}\right) l_{1}+a_{12} \kappa_{2} l_{2}\right]}{\left(1-a_{11} \kappa_{2}\right)\left(1-b_{12} \kappa_{2}\right)-a_{12} b_{11} \kappa_{2}^{2}}, \\
& \int_{1}^{e} v(t) \phi(t) \frac{d t}{t} \leq \frac{\left(\kappa_{2} \Gamma(\alpha) / \Gamma(\alpha+2)\right)\left[b_{11} \kappa_{2} l_{1}+\left(1-a_{11} \kappa_{2}\right) l_{2}\right]}{\left(1-a_{11} \kappa_{2}\right)\left(1-b_{12} \kappa_{2}\right)-a_{12} b_{11} \kappa_{2}^{2}} .
\end{aligned}
$$

Note that $u, v \in P_{0}$, we have

$$
\begin{gathered}
\|u\| \leq \frac{\left(\omega_{0}^{-1} \kappa_{2} \Gamma(\alpha) / \Gamma(\alpha+2)\right)\left[\left(1-b_{12} \kappa_{2}\right) l_{1}+a_{12} \kappa_{2} l_{2}\right]}{\left(1-a_{11} \kappa_{2}\right)\left(1-b_{12} \kappa_{2}\right)-a_{12} b_{11} \kappa_{2}^{2}}, \\
\|v\| \leq \frac{\left(\omega_{0}^{-1} \kappa_{2} \Gamma(\alpha) / \Gamma(\alpha+2)\right)\left[b_{11} \kappa_{2} l_{1}+\left(1-a_{11} \kappa_{2}\right) l_{2}\right]}{\left(1-a_{11} \kappa_{2}\right)\left(1-b_{12} \kappa_{2}\right)-a_{12} b_{11} \kappa_{2}^{2}} .
\end{gathered}
$$

Taking $R_{2}>\left(\omega_{0}^{-1} \kappa_{2} \Gamma(\alpha) / \Gamma(\alpha+2)\right)\left[\left(1-b_{12} \kappa_{2}\right) l_{1}+a_{12} \kappa_{2} l_{2}+\right.$ $\left.b_{11} \kappa_{2} l_{1}+\left(1-a_{11} \kappa_{2}\right) l_{2}\right] /\left(1-a_{11} \kappa_{2}\right)\left(1-b_{12} \kappa_{2}\right)-a_{12} b_{11} \kappa_{2}^{2}$ and $R_{2}>r_{2}\left(r_{2}\right.$ is defined by $\left.(\mathrm{H} 4)\right)$, we have

$(u, v) \neq \mu A(u, v), \quad$ for $(u, v) \in \partial B_{R_{2}} \cap(P \times P), \mu \in[0,1]$.

Then, Lemma 7 enables us to obtain

$$
i\left(A, B_{R_{2}} \cap(P \times P), P \times P\right)=1 .
$$

From (66) and (76), we have

$$
\begin{aligned}
& i\left(A,\left(B_{R_{2}} \backslash \bar{B}_{r_{2}}\right) \cap(P \times P), P \times P\right) \\
& =i\left(A, B_{R_{2}} \cap(P \times P), P \times P\right)-i\left(A, B_{r_{2}} \cap(P \times P), P \times P\right) \\
& =1-0=1 .
\end{aligned}
$$

Therefore, the operator $A$ has at least one fixed point on $\left(B_{R_{2}} \backslash \bar{B}_{r_{2}}\right) \cap(P \times P)$. Equivalently, (1) has at least one positive solution. This completes the proof.

In (1), let $n=3, \alpha=2.5$, and $h(t)=\log t, t \in[1, e]$. Then, $\int_{1}^{e} h(t)(\log t)^{\alpha-1}(d t / t)=\int_{1}^{e}(\log t)^{\alpha}(d t / t)=(2 / 7) \in[0,1)$ and (H0) holds. Moreover, we can calculate $\mathscr{K}, \kappa_{1}$, and $\kappa_{2}$ as follows:

$$
\begin{aligned}
& \mathscr{K}=\frac{\alpha-1}{\Gamma(\alpha)}\left[1+\frac{\int_{1}^{e} h(t)(d t / t)}{1-\int_{1}^{e} h(t)(\log t)^{\alpha-1}(d t / t)}\right]=\frac{1.5}{\Gamma(2.5)}\left[1+\frac{\int_{1}^{e}(\log t)(d t / t)}{1-\int_{1}^{e}(\log t)^{2.5}(d t / t)}\right] \approx 1.92, \\
& \kappa_{1}=\frac{\alpha^{2} \Gamma(\alpha)}{\Gamma(2 \alpha+2)}+\frac{\Gamma(\alpha)}{2 \Gamma(2 \alpha)} \frac{\int_{1}^{e} h(t)(\log t)^{\alpha-1}(1-\log t)(d t / t)}{1-\int_{1}^{e} h(t)(\log t)^{\alpha-1}(d t / t)}=\frac{(2.5)^{2} \Gamma(2.5)}{\Gamma(7)}+\frac{\Gamma(2.5)}{2 \Gamma(5)} \frac{\Gamma(3.5)}{\Gamma(5.5)} \frac{7}{5} \approx 0.014, \\
& \kappa_{2}=\frac{\alpha-1}{\Gamma(\alpha+2)}\left[1+\frac{\int_{1}^{e} h(t)(d t / t)}{1-\int_{1}^{e} h(t)(\log t)^{\alpha-1}(d t / t)}\right]=\frac{1.5}{\Gamma(4.5)}\left[1+\frac{\int_{1}^{e}(\log t)(d t / t)}{1-\int_{1}^{e}(\log t)^{2.5}(d t / t)}\right] \approx 0.22 .
\end{aligned}
$$

Example 1. Let $f_{1}(t, u, v)=(u+v)^{\gamma_{1}}, f_{2}(t, u, v)=(u+v)^{\gamma_{2}}$, $p_{1}(v)=v^{1 / 3}, q_{1}(u)=u^{4}, p_{2}(v)=v^{2}$, and $q_{2}(u)=u$, for $(t, u, v) \in$ $[1, e] \times \mathbb{R}^{+} \times \mathbb{R}^{+}$, where $\gamma_{1}>2$ and $\gamma_{2}>4$. Then, we have

(i) $\lim \inf _{v \longrightarrow+\infty}\left(f_{1}(t, u, v) / p_{1}(v)\right)=\liminf _{v \longrightarrow+\infty}$ $\left((u+v)^{\gamma_{1}} / v^{1 / 3}\right) \geq \lim \inf _{v \longrightarrow+\infty}\left(v^{\gamma_{1}} / v^{1 / 3}\right)=+\infty$, for all $(t, u) \in[1, e] \times \mathbb{R}^{+}$

(ii) $\lim \inf _{u \longrightarrow+\infty}\left(f_{2}(t, u, v) / q_{1}(u)\right)=\liminf _{u \longrightarrow+\infty}$ $\left((u+v)^{\gamma_{2}} / u^{4}\right) \geq \lim \inf _{u \longrightarrow+\infty}\left(u^{\gamma_{2}} / u^{4}\right)=+\infty, \quad$ for all $(t, v) \in[1, e] \times \mathbb{R}^{+}$

(iii) $\lim \sup _{u+v \longrightarrow 0^{+}}\left(f_{1}(t, u, v) / p_{2}(v)\right)=\lim \sup _{u+v \longrightarrow 0^{+}}$ $\left((u+v)^{\gamma_{1}} / v^{2}\right)=0$, for all $t \in[1, e]$

(iv) $\lim \sup _{u+v \longrightarrow 0^{+}}\left(f_{2}(t, u, v) / q_{2}(u)\right)=\lim \sup _{u+v \longrightarrow 0^{+}}$ $\left((u+v)^{\gamma_{2}} / u\right)=0$, for all $t \in[1, e]$

(v) $\lim \inf _{z \longrightarrow+\infty}\left(p_{1}\left(\mathscr{K} q_{1}(z)\right) / z\right)=\lim _{z \rightarrow+\infty}$ $\left(\sqrt[3]{\mathscr{K}} z^{4 / 3} / z\right)=+\infty$ (vi) $\lim \sup _{z \longrightarrow 0^{+}}\left(p_{2}\left(\mathscr{K} q_{2}(z)\right) / z\right)=\lim \sup _{z \longrightarrow 0^{+}}\left(\mathscr{K}^{2} z^{2} /\right.$ $z)=0$

Therefore, (H2)-(H3) hold.

Example 2. Let $a_{11}=0.05, a_{12}=0.6, b_{11}=0.4$, and $b_{12}=0.08$, then we calculate $a_{11} \kappa_{2}=0.011<1, b_{12} \kappa_{2}=$ $0.0176<1$, and

$$
\left|\begin{array}{cc}
1-a_{11} \kappa_{2} & -a_{12} \kappa_{2} \\
-b_{11} \kappa_{2} & 1-b_{12} \kappa_{2}
\end{array}\right|=\left|\begin{array}{cc}
0.989 & -0.132 \\
-0.088 & 0.9824
\end{array}\right| \approx 0.96 .
$$

Let $\quad f_{1}(t, u, v)=\left(a_{11} u+a_{12} v\right)^{\gamma_{3}}, f_{2}(t, u, v)=\left(b_{11} u+\right.$ $\left.b_{12} v\right)^{\gamma_{4}}, p_{3}(v)=\sqrt{v}$, and $q_{3}(u)=u^{3 / 4}$, for $(t, u, v) \in[1, e] \times$ $\mathbb{R}^{+} \times \mathbb{R}^{+}$, where $\gamma_{3} \in(0,(1 / 2))$ and $\gamma_{4} \in(0,(3 / 4))$. Then for all $t \in[1, e]$, we have 


$$
\begin{aligned}
\liminf _{a_{11} u+a_{12} v \longrightarrow 0^{+}} \frac{f_{1}(t, u, v)}{p_{3}(v)}=\liminf _{a_{11} u+a_{12} v \longrightarrow 0^{+}} \frac{\left(a_{11} u+a_{12} v\right)^{\gamma_{3}}}{v^{1 / 2}} \geq \liminf _{a_{11} u+a_{12} v \longrightarrow 0^{+}} \frac{\left(a_{12} v\right)^{\gamma_{3}}}{v^{1 / 2}}=+\infty, \\
\liminf _{b_{11} u+b_{12} v \longrightarrow 0^{+}} \frac{f_{2}(t, u, v)}{q_{3}(u)}=\liminf _{b_{11} u+b_{12} v \longrightarrow 0^{+}} \frac{\left(b_{11} u+b_{12} v\right)^{\gamma_{4}}}{u^{3 / 4}} \geq \liminf _{b_{11} u+b_{12} v \longrightarrow 0^{+}} \frac{\left(b_{11} u\right)^{\gamma_{4}}}{u^{3 / 4}}=+\infty, \\
\limsup _{a_{11} u+a_{12} v \longrightarrow+\infty} \frac{f_{1}(t, u, v)}{a_{11} u+a_{12} v}=\limsup _{a_{11} u+a_{12} v \longrightarrow+\infty} \frac{\left(a_{11} u+a_{12} v\right)^{\gamma_{3}}}{a_{11} u+a_{12} v}=0, \\
\limsup _{b_{11} u+b_{12} v \longrightarrow+\infty} \frac{f_{2}(t, u, v)}{b_{11} u+b_{12} v}=\limsup _{b_{11} u+b_{12} v \longrightarrow+\infty} \frac{\left(b_{11} u+b_{12} v\right)^{\gamma_{4}}}{b_{11} u+b_{12} v}=0, \\
\liminf _{z \longrightarrow 0^{+}} \frac{p_{3}\left(\mathscr{K} q_{3}(z)\right)}{z}=\liminf _{z \longrightarrow 0^{+}} \frac{\sqrt{\mathscr{K}} z^{3 / 8}}{z}=+\infty .
\end{aligned}
$$

As a result, (H4)-(H5) hold.

\section{Data Availability}

No data were used to support this study.

\section{Conflicts of Interest}

The authors declare that there are no conflicts of interest regarding the publication of this paper.

\section{Acknowledgments}

This work was supported by the National Natural Science Foundation of China (Grant no. 11601048), University Natural Science Foundation of Anhui Provincial Education Department (Nos. KJ2017A442 and KJ2018A0452), and Natural Science Foundation of Chongqing Normal University (Grant no. 16XYY24).

\section{References}

[1] C. Yuan, "Multiple positive solutions for $(n-1,1)$-type semipositone conjugate boundary value problems of nonlinear fractional differential equations," Electronic Journal of Qualitative Theory of Differential Equations, vol. 36, no. 36, pp. 1-12, 2010.

[2] Y. Cui, "Uniqueness of solution for boundary value problems for fractional differential equations," Applied Mathematics Letters, vol. 51, pp. 48-54, 2016.

[3] Y. Cu, W. Ma, and Q. Sun, "New uniqueness results for boundary value problem of fractional differential equation," Nonlinear Analysis: Modelling and Control, vol. 23, no. 1, pp. 31-39, 2018.

[4] Y. Zou and G. He, "On the uniqueness of solutions for a class of fractional differential equations," Applied Mathematics Letters, vol. 74, pp. 68-73, 2017.

[5] Z. Fu, S. Bai, D. O’Regan, and J. Xu, "Nontrivial solutions for an integral boundary value problem involving RiemannLiouville fractional derivatives," Journal of Inequalities and Applications, vol. 2019, Article ID 104, 16 pages, 2019.

[6] X. Zhang, J. Wu, L. Liu, Y. Wu, and Y. Cui, "Convergence analysis of iterative scheme and error estimation of positive solution for a fractional differential equation," Mathematical Modelling and Analysis, vol. 23, no. 4, pp. 611-626, 2018.

[7] S. Meng and Y. Cui, "Multiplicity results to a conformable fractional differential equations involving integral boundary condition," Complexity, vol. 2019, Article ID 8402347, 8 pages, 2019.

[8] C. Zhai, P. Li, and H. Li, "Single upper-solution or lowersolution method for Langevin equations with two fractional orders," Advances in Difference Equations, vol. 2018, Article ID 360, 10 pages, 2018.

[9] X. Dong, Z. Bai, and S. Zhang, "Positive solutions to boundary value problems of $p$-Laplacian with fractional derivative," Boundary Value Problems, vol. 2017, Article ID 5, 15 pages, 2017.

[10] Q. Song and Z. Bai, "Positive solutions of fractional differential equations involving the Riemann-Stieltjes integral boundary condition," Advances in Difference Equations, vol. 2018, Article ID 183, 7 pages, 2018.

[11] Y. Zou and G. He, "The existence of solutions to integral boundary value problems of fractional differential equations at resonance," Journal of Function Spaces, vol. 2017, Article ID 2785937, 7 pages, 2017.

[12] W. Ma, S. Meng, and Y. Cui, "Resonant integral boundary value problems for Caputo fractional differential equations," Mathematical Problems in Engineering, vol. 2018, Article ID 5438592, 8 pages, 2018.

[13] M. Zuo, X. Hao, L. Liu, and Y. Cui, "Existence results for impulsive fractional integro-differential equation of mixed type with constant coefficient and antiperiodic boundary conditions," Boundary Value Problems, vol. 2017, Article ID 161, 15 pages, 2017.

[14] Q. Song, X. Dong, Z. Bai, and B. Chen, "Existence for fractional Dirichlet boundary value problem under barrier strip conditions," The Journal of Nonlinear Sciences and Applications, vol. 10, no. 7, pp. 3592-3598, 2017.

[15] Z. Bai, X. Dong, and C. Yin, "Existence results for impulsive nonlinear fractional differential equation with mixed boundary conditions," Boundary Value Problems, vol. 2016, Article ID 63, 11 pages, 2016.

[16] Z. Bai, Y. Chen, H. Lian, and S. Sun, "On the existence of blow up solutions for a class of fractional differential equations," Fractional Calculus and Applied Analysis, vol. 17, no. 4, pp. 1175-1187, 2014.

[17] K. Sheng, W. Zhang, and Z. Bai, "Positive solutions to fractional boundary-value problems with $p$-Laplacian on time 
scales," Boundary Value Problems, vol. 2018, Article ID 70, 15 pages, 2018.

[18] P. Wang and X. Liu, "Rapid convergence for telegraph systems with periodic boundary conditions," Journal of Function Spaces, vol. 2017, Article ID 1982568, 10 pages, 2017.

[19] K. Zhang, J. Wang, and W. Ma, "Solutions for integral boundary value problems of nonlinear Hadamard fractional differential equations," Journal of Function Spaces, vol. 2018, Article ID 2193234, 10 pages, 2018.

[20] K. Zhang and Z. Fu, "Solutions for a class of Hadamard fractional boundary value problems with sign-changing nonlinearity," Journal of Function Spaces, vol. 2019, Article ID 9046472, 7 pages, 2019.

[21] C. Zhai, W. Wang, and H. Li, "A uniqueness method to a new Hadamard fractional differential system with four-point boundary conditions," Journal of Inequalities and Applications, vol. 2018, Article ID 207, 16 pages, 2018.

[22] W. Yang, "Positive solutions for singular coupled integral boundary value problems of nonlinear Hadamard fractional differential equations," Journal of Nonlinear Sciences and Applications, vol. 08, no. 2, pp. 110-129, 2015.

[23] T. Qi, Y. Liu, and Y. Zou, "Existence result for a class of coupled fractional differential systems with integral boundary value conditions," The Journal of Nonlinear Sciences and Applications, vol. 10, no. 7, pp. 4034-4045, 2017.

[24] T. Qi, Y. Liu, and Y. Cui, "Existence of solutions for a class of coupled fractional differential systems with nonlocal boundary conditions," Journal of Function Spaces, vol. 2017, Article ID 6703860, 9 pages, 2017.

[25] Y. Zhao, X. Hou, Y. Sun, and Z. Bai, "Solvability for some class of multi-order nonlinear fractional systems," Advances in Difference Equations, vol. 2019, Article ID 23, 10 pages, 2019.

[26] Y. Zhang, "Existence results for a coupled system of nonlinear fractional multi-point boundary value problems at resonance," Journal of Inequalities and Applications, vol. 2018, Article ID 198, 17 pages, 2018.

[27] H. Li and J. Zhang, "Positive solutions for a system of fractional differential equations with two parameters," Journal of Function Spaces, vol. 2018, Article ID 1462505, 9 pages, 2018.

[28] X. Zhang, L. Liu, Y. Wu, and Y. Zou, "Existence and uniqueness of solutions for systems of fractional differential equations with Riemann-Stieltjes integral boundary condition," Advances in Difference Equations, vol. 2018, Article ID 204, 15 pages, 2018.

[29] X. Hao, H. Wang, L. Liu, and Y. Cui, "Positive solutions for a system of nonlinear fractional nonlocal boundary value problems with parameters and p-Laplacian operator," Boundary Value Problems, vol. 2017, Article ID 182, 18 pages, 2017.

[30] W. Cheng, J. Xu, and Y. Cui, "Positive solutions for a system of nonlinear semipositone fractional q q-difference equations with q q-integral boundary conditions," The Journal of Nonlinear Sciences and Applications, vol. 10, no. 8, pp. 4430-4440, 2017.

[31] U. Riaz, A. Zada, Z. Ali, Y. Cui, and J. Xu, "Analysis of coupled systems of implicit impulsive fractional differential equations involving Hadamard derivatives," Advances in Difference Equations, vol. 2019, Article ID 226, 27 pages, 2019.

[32] U. Riaz, A. Zada, Z. Ali, M. Ahmad, J. Xu, and Z. Fu, “Analysis of nonlinear coupled systems of impulsive fractional differential equations with Hadamard derivatives," Mathematical Problems in Engineering, vol. 2019, Article ID 5093572, 20 pages, 2019.
[33] A. A. Kilbas, H. M. Srivastava, and J. J. Trujillo, Theory and Applications of Fractional Differential Equations, Elsevier, Boston, MA, USA, 2006.

[34] D. Guo and V. Lakshmikantham, Nonlinear Problems in Abstract Cones, Academic Press, Orlando, FL, USA, 1988. 


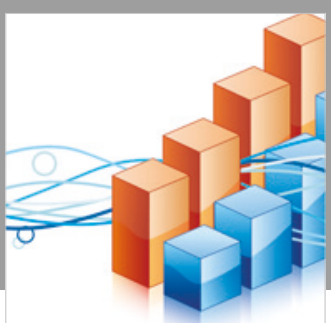

Advances in

Operations Research

\section{-n-m}
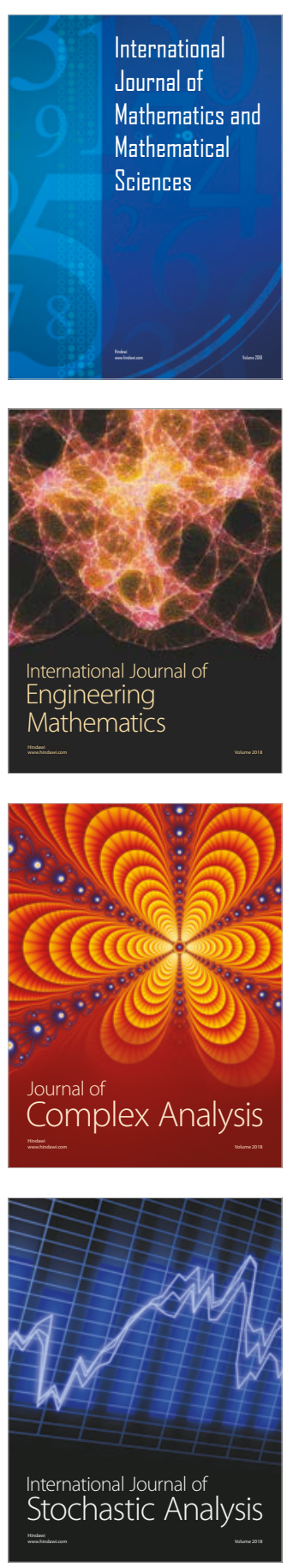
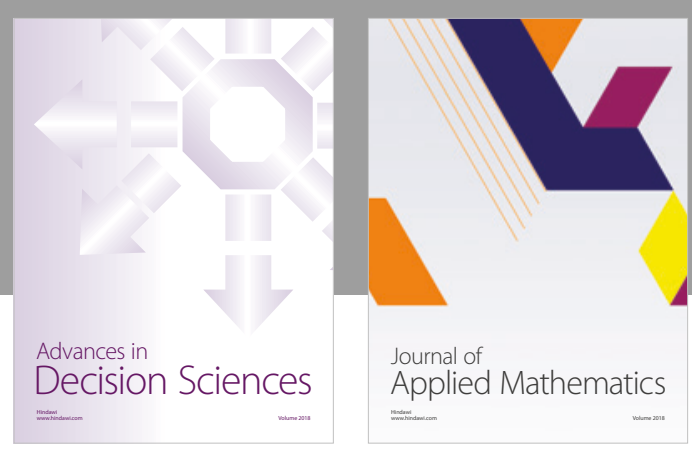

Journal of

Applied Mathematics
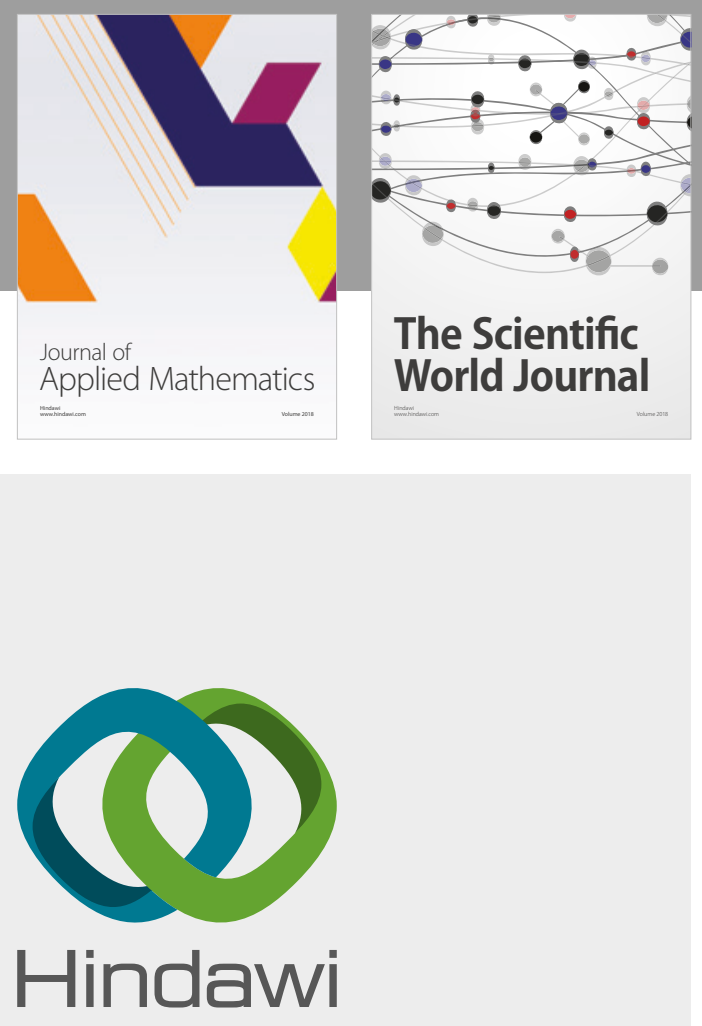

Submit your manuscripts at

www.hindawi.com

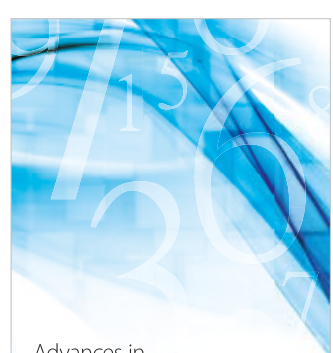

Advances in
Numerical Analysis
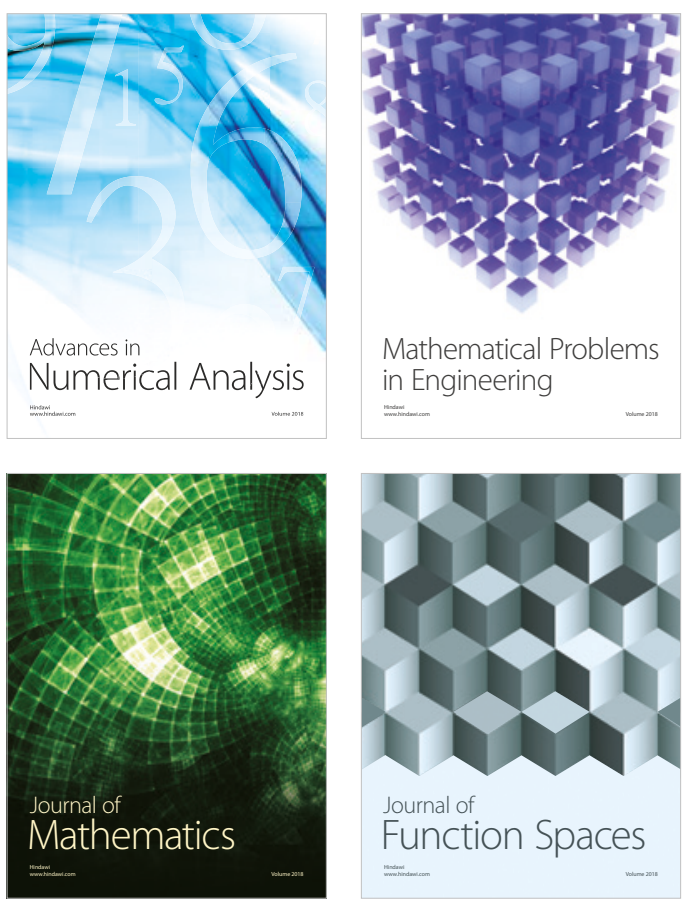

Mathematical Problems in Engineering

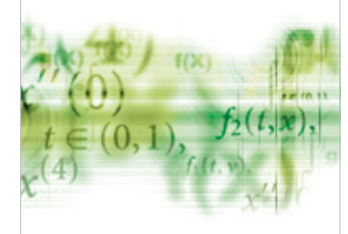

International Journal of

Differential Equations

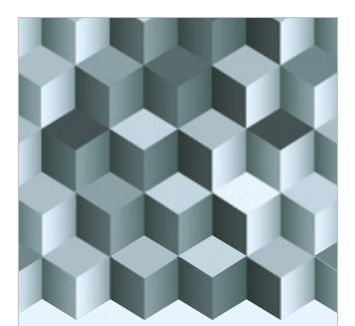

Journal of

Function Spaces
The Scientific

World Journal

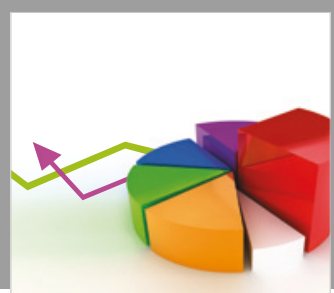

Journal of

Probability and Statistics
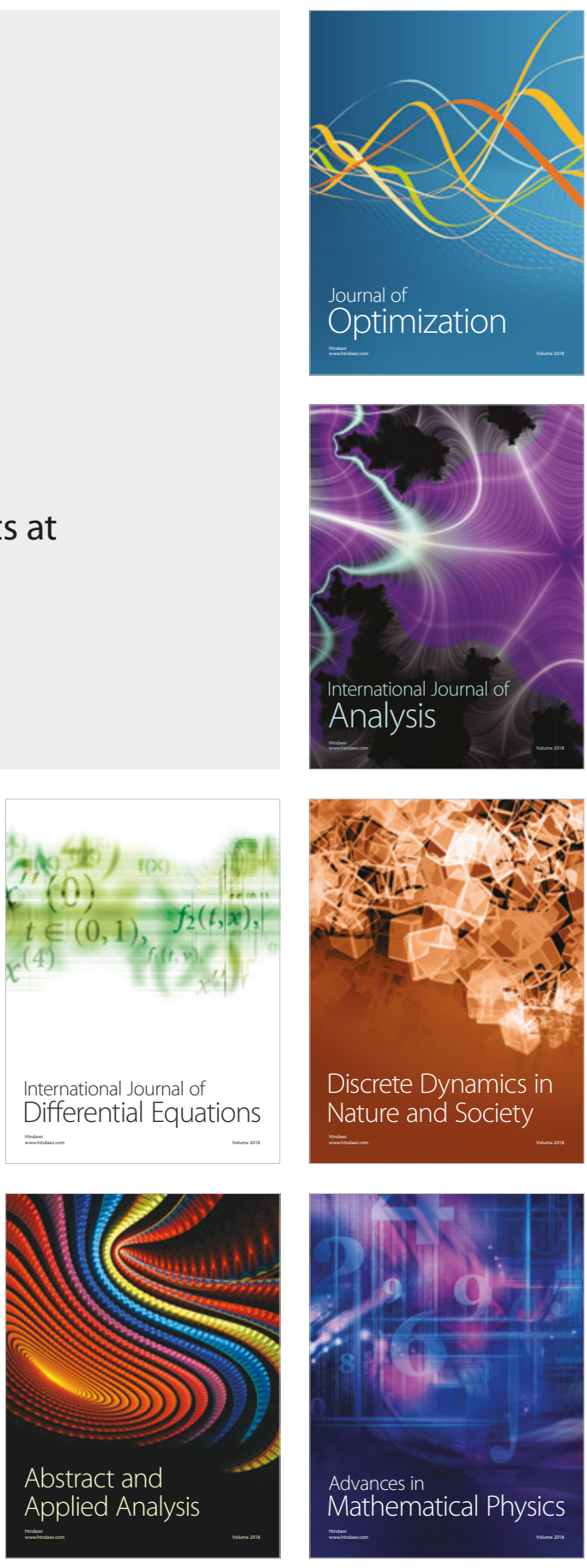